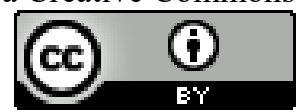

\title{
A EDUCAÇÃO DA TEORIA À PRÁXIS: ONDE ESTÃO AS RELIGIÕES AFRO-BRASILEIRAS?
}

Yuri Miguel Macedo ${ }^{1}$

Resumo: As religiões afro-brasileiras ainda carregam o peso social da exclusão e intolerância, justamente por serem pautadas e fundamentadas sob o continente africano que historicamente, pelo processo de colonização, foi forjado a abdicar de sua cultura e seus saberes. Nesse sentido, o presente trabalho busca levantar e apresentar dados de dissertações e teses produzidas entre os séculos XX e XXI retratadas no lapso temporal de 1990 a 2018, que contemplem temáticas que girem em torno das religiões afrobrasileiras.

Palavras-Chave: Religiões afro-brasileiras; Educação; Dissertações e teses.

\section{EDUCATION OF THEORY TO PRAXIS: WHERE ARE AFRO-BRAZILIAN RELIGIONS?}

Abstract: Afro-Brazilian religions still carry the social weight of exclusion and intolerance, precisely because they are guided and grounded under the African continent that, historically, through the colonization process, was forged to abdicate its culture and knowledge. In this sense, the present work seeks to raise and present data from dissertations and theses produced between the 20th and 21 st centuries portrayed in the period of time from 1990 to 2018, which contemplate themes that revolve around AfroBrazilian religions.

Keywords: Afro-Brazilian religions; Education; Dissertations and theses.

\section{EDUCACIÓN DE LA TEORÍA A LA PRAXIS: ¿DÓNDE ESTÁN LAS RELIGIONES AFROBRASILEÑAS?}

Resumen: Las religiones afrobrasileñas aún cargan con el peso social de la exclusión y la intolerancia, precisamente porque están guiadas y arraigadas bajo el continente africano que, históricamente, debido al proceso de colonización, se vio obligado a abdicar de su cultura y conocimientos. En este sentido, el presente trabajo busca levantar y presentar

\footnotetext{
${ }^{1}$ Professor Pesquisador do Núcleo de Estudos Afro-Brasileiros da Universidade Federal do Espirito Santo (UFES), Professor no Programa de Pós-Graduação Lato Sensu Formação de Professores em Letras-Libras na Universidade do Estado da Bahia (UNEB), Aluno do Programa de Pós-Graduação em Ensino e Relações Étnico-Raciais da Universidade Federal do Sul da Bahia (UFSB). ORCID: https://orcid.org/0000-00030926-6553. E-mail: yurimacedo@id.uff.br
} 
datos de disertaciones y tesis producidas entre los siglos XX y XXI retratadas en el período de tiempo de 1990 a 2018, que contemplan temas que giran en torno a las religiones afrobrasileñas.

Palabras-clave: Religiones afrobrasileñas; Educación; Disertaciones y tesis.

\section{ÉDUCATION DE LA THÉORIE À LA PRAXIS: O SONT LES RELIGIONS AFRO-BRÉSILIENNES?}

Résumé: Les religions afro-brésiliennes portent toujours le poids social de l'exclusion et de l'intolérance, précisément parce qu'elles sont guidées et ancrées dans le continent africain qui, historiquement, en raison du processus de colonisation, a été contraint d'abdiquer sa culture et ses connaissances. En ce sens, le présent travail cherche à collecter et à présenter des données à partir de dissertations et de thèses produites entre les XXe et XXIe siècles dépeints entre 1990 et 2018, qui incluent des thèmes qui tournent autour des religions afro-brésiliennes.

Mots-clés: Religions afro-brésiliennes; Éducation; Dissertations et thèses.

\section{ÀBI ${ }^{2}$}

O presente texto apresenta o levantamento das dissertações e teses que tratam, especificamente, das religiões afro-brasileiras, de modo que foi usado para busca no Catálogo de Teses e Dissertações da Coordenação de Aperfeiçoamento de Pessoal de Nível Superior (CAPES) nos seguintes termos: religiões afro-brasileiras, candomblé e umbanda. Termos esses que representam a área afim dos estudos referentes às religiões de matrizes africanas. A demanda por essa pesquisa se alicerça no reconhecimento da atual, e contínua, situação de perseguição, intolerância e exclusão dos(as) adeptos(as) das religiões afro-brasileiras, ou seja, as religiões não adeptas veem as religiões de matrizes africanas como demoníacas.

Nesse sentido, é necessário rever os conceitos de religiões apresentados no século XXI, tomando por base as suas criações e adeptos em todo mundo. O levantamento trará a coleção em 2 partes, dissertações e teses do século XX (janeiro de 1990 - dezembro de 2000) e teses do século XXI (janeiro de 2001 - dezembro de 2018), que será analisado como a demanda de produções científicas nas defesas de dissertações e teses, e se foi crescente ou decrescente aos estudos voltado as Religiões Afro-Brasileira.

\footnotetext{
${ }^{2}$ Palavra iorubá que significa nascer.
} 
Faz-se necessário a reflexão dessa temática tendo em vista os inúmeros casos de intolerância religiosa que tem crescido assustadoramente no Brasil, sendo um país, constitucionalmente laico. E diante de várias evidências científicas, podemos ver que a Educação é uma das instituições que nos ajuda na formação de cidadãos que respeitem a dignidade, diferença, individualidade, subjetividade e escolhas. MACEDO et al., (2019) corrobora com a seguinte afirmação "acreditamos ser a educação, a área de conhecimento fundamental para fissurar tais estruturas" mais quais seriam as estruturas que precisam ser fissuradas?

As Universidades e pincipalmente os Programas de Pós-graduação precisam se consolidar nos espaços de produção de conhecimento dessa temática que por hora está sendo um dos maiores problemas nos processos sociais que envolvem as questões de religião e religiosidade.

Macedo et al. (2019), sinaliza que a:

"[...]sociedade moderna foi caracterizada por produções de binômios, tais como: riqueza e pobreza, homem e mulher e branco e negro. Chegamos ao século XXI e, como resultado desta sociedade, marcadamente ocidental e com traços históricos de colonialismos, podemos encontrar a humanidade com condutas que levam a extremos diferentes como se fossem uma ruptura. Se por um lado pareça assustador, por outro não se pode negar os progressos alcançados, consequentes da lógica capitalista" (p. 204).

A partir desses binômios e desde a construção histórica do país, os exescravizados $^{3}$, perderam seus direitos culturais e religiosos assim que chegaram a Costa Brasileira, cultura essa que existe bem antes do surgimento do cristianismo no mundo.

Olhar para essa religiosidade afro-brasileira e para as religiões de matrizes africanas, é tirar essas religiões do espaço do periférico e/ou marginalizado. Eé necessário demarcar que os estudos na temática proposta, têm sido cada vez mais temas de pesquisas, por um viés decolonial que se acredita que "urge a necessidade de protagonizar novas posições com vistas à legitimação e reconhecimento de outros povos, de outras formas de ensinar e de aprender" (Santos; Valadares; Macedo, 2019, p. 207). A partir das teses que foram catalogadas, percebemos que as temáticas relacionadas às religiões afro-brasileiras:

\footnotetext{
3 “Utilizaremos a palavra ex-escravizado, ao invés de escravo, mais uma vez por acreditar na força política das palavras" (Passos, 2019).
} 
candomblé e umbanda são uma demanda crescente do século XXI em relação ao século XX de uma porcentagem de $1900 \%$.

\section{MOJÚ 4}

\section{DISSERTAÇÕES DO SÉCULO XX}

RAMOS, WELLINGTON SANTOS. SIGNIFICADO DO SACRIFÍCIO NO CANDOMBLÉ' 01/12/1999 141 f. Mestrado em CIÊNCIAS DA RELIGIÃO Instituição de Ensino: PONTIFíCIA UNIVERSIDADE CATÓLICA DE SÃO PAULO, SÃO PAULO.

PASSOS, MARA DE SÁ MARTINS DA COSTA. EXU PEDE PASSAGEM ' 01/08/1999 $200 \mathrm{f}$. Mestrado em CIÊNCIAS DA RELIGIÃO Instituição de Ensino: PONTIFÍCIA UNIVERSIDADE CATÓLICA DE SÃO PAULO, SÃO PAULO.

PIRES, ALVARO ROBERTO. A ADOLESCENTE, A MULHER E IANSÃ. ESTUdO DO IMAGINÁRIO NA PASSAGEM DA INFÂNCIA PARA A ADOLESCENCIA NO INTERIOR DO ACHE ILE OBA.' 01/10/1993 136 f. Mestrado em CIÊNCIAS SOCIAIS Instituição de Ensino: PONTIFÍCIA UNIVERSIDADE CATÓLICA DE SÃO PAULO.

BOCCHI, POLLATO SILVANA ELVIRA. O FASCÍNIO DO CANDOMBLÉ PAULISTA: SEUS CLIENTES E UM OLUO.' 01/08/1990 239 f. Mestrado em CIÊNCIAS SOCIAIS Instituição de Ensino: PONTIFÍCIA UNIVERSIDADE CATÓLICA DE SÃO PAULO, SÃO PAULO.

NATALINO, GERALDO JOSÉ. CANDOMBLÉ E CRISTIANISMO: DUAS MISTAGOGIAS EM CONFRONTO' 23/02/1999 170 f. Mestrado em TEOLOGIA Instituição de Ensino: PONTIFÍCIA UNIVERSIDADE CATÓLICA DO RIO DE JANEIRO, Rio de Janeiro.

AGUIAR, ITAMAR PEREIRA DE. AS RELIGIÕES AFRO-BRASILEIRAS EM VITÓRIA DA CONQUISTA: CAMINHOS DA DIVERSIDADE.' 01/09/1999 201 f. Mestrado em CIÊNCIAS SOCIAIS Instituição de Ensino: PONTIFÍCIA UNIVERSIDADE CATÓLICA DE SÃO PAULO, SÃO PAULO.

RODRIGUES, VALERIO. DIVINO MARAVILHOSO: PRÁTICAS ARTÍSTICAS E TRADIÇÃO CULTURAL NOS CANDOMBLÉS DO RIO DE JANEIRO' 01/03/1999 218 f. Mestrado em ARTES VISUAIS Instituição de Ensino: UNIVERSIDADE FEDERAL DO RIO DE JANEIRO, RIO DE JANEIRO.

MESQUITA, RALPH RIBEIRO. SIGNIFICAÇÃO DAS RELAÇÕES DE PODER E AUTORIDADE EM COMUNIDADES RELIGIOSAS AFRO-BRASILEIRAS DE ORIGEM "NAGO"' 01/09/1995 340 f. Mestrado em PSICOLOGIA Instituição de Ensino: UNIVERSIDADE DO ESTADO DO RIO DE JANEIRO, RIO DE JANEIRO.

SANTIAGO, JAIRO DA COSTA. CANDOMBLÉ, COMUNICAÇÃO E MITO: A SOBREVIVÊNCIA DA RELIGIOSIDADE AFRO-LUSO-BRASILEIRA' 01/09/1999 123 f. Mestrado em COMUNICAÇÃO Instituição de Ensino: UNIVERSIDADE FEDERAL DO RIO DE JANEIRO, RIO DE JANEIRO.

ANDRADE, ANA BEATRIZ PEREIRA DE. ENCRUZILHADA DE SÍMBOLOS: UM OLHAR SOBRE OS OBJETOS SAGRADOS E O CONSUMO NO CANDOMBLÉ DO RIO DE JANEIRO' 01/03/1999 113 f. Mestrado em COMUNICAÇÃO Instituição de Ensino: UNIVERSIDADE FEDERAL DO RIO DE JANEIRO, RIO DE JANEIRO.

\footnotetext{
${ }^{4}$ Palavra em iorubá que significa conhecer.
} 
SANTOS, JOCELIO TELLES. O DONO DA TERRA - A PRESENÇA DO CABOCLO NO CANDOMBLÉ BAIANO' 01/08/1992 170 f. Mestrado em CIÊNCIA SOCIAL (ANTROPOLOGIA SOCIAL) Instituição de Ensino: UNIVERSIDADE DE SÃO PAULO, São Paulo.

AQUINO, FONSECA EDUARDO PACHECO DE. O CANDOMBLÉ E A DANCA DA VIDA: AFLIÇÃO, CURA E AFILIAÇÃo RELIGIOSA AO PALÁCIO DE YEMANJA' 01/12/1995 149 f. Mestrado em ANTROPOLOGIA Instituição de Ensino: UNIVERSIDADE FEDERAL DE PERNAMBUCO, RECIFE.

CRUZ, ROBSON ROGERIO. CARREGO DE EGUM - CONTRIBUIÇÃO AOS ESTUDOS DO RITO MORTUÁRIO NO CANDOMBLÉ. 01/11/1995 100 f. Mestrado em ANTROPOLOGIA SOCIAL Instituição de Ensino: UNIVERSIDADE FEDERAL DO RIO DE JANEIRO, RIO DE JANEIRO.

LIMA, NELSON. "DANDO CONTA DO RECADO: A DANÇA AFRO NO RIO DE JANEIRO E SUAS INFLUÊNCIAS."' 01/11/1995 94 f. Mestrado em SOCIOLOGIA E ANTROPOLOGIA Instituição de Ensino: UNIVERSIDADE FEDERAL DO RIO DE JANEIRO, RIO DE JANEIRO.

SILVA, VAGNER GONCALVES DA. O CANDOMBLÉ NA CIDADE. TRADIÇÃO E RENOVAÇÃOO' 01/11/1992 282 f. Mestrado em CIÊNCIA SOCIAL (ANTROPOLOGIA SOCIAL) Instituição de Ensino: UNIVERSIDADE DE SÃO PAULO, São Paulo.

NETO, JOSE MARINHO DOS SANTOS. OBSSESSÃO E MODERNIDADE UM ESTUDO DE CASO SOBRE CULTOS AFRO-BRASILEIROS EM BRASÍLIA.' 01/09/1993 275 f. Mestrado em ANTROPOLOGIA Instituição de Ensino: UNIVERSIDADE DE BRASÍLIA, BRASÍLIA.

GONCALVES, MARIA ALICE REZENDE. A BRINCADEIRA NO TERREIRO DE OXOSSI: UM ESTUDO SOBRE A VIDA LÚDICA DE UMA COMUNIDADE DE CANDOMBLÉ DO GRANDE RIO.' 01/12/1990 259 f. Mestrado em SOCIOLOGIA E ANTROPOLOGIA Instituição de Ensino: UNIVERSIDADE FEDERAL DO RIO DE JANEIRO, RIO DE JANEIRO.

SOARES, MARIZA DE CARVALHO. O MEDO DA VIDA E O MEDO DA MORTE UM ESTUDO DA RELIGIOSIDADE BRASILEIRA' 01/12/1990 163 f. Mestrado em ANTROPOLOGIA SOCIAL Instituição de Ensino: UNIVERSIDADE FEDERAL DO RIO DE JANEIRO, RIO DE JANEIRO.

CAPONE, STEFANIA. A DANÇA DOS DEUSES: UMA ANÁLISE DA DANÇA DE POSSESSÃO NO CANDOMBLÉ AN-GOLA KASSANJE".' 01/03/1991 289 f. Mestrado em ANTROPOLOGIA SOCIAL Instituição de Ensino: UNIVERSIDADE FEDERAL DO RIO DE JANEIRO, RIO DE JANEIRO.

LARA, LARISSA MICHELE. AS DANÇAS DO SAGRADO PROFANO: TRANSPONDO TEMPOS E ESPAÇOS EM RITUAIS DO CANDOMBLÉ.' 01/08/1999 206 f. Mestrado em EDUCAÇÃO FÍSICA Instituição de Ensino: UNIVERSIDADE ESTADUAL DE CAMPINAS, CAMPINAS.

AMARAL, RITA DE CASSIA DE M. P.. POVO DE SANTO, POVO DE FESTA. ESTUdO ANTROPOLÓGICO DO ESTILO DE VIDA DOS ADEPTOS DO CANDOMBLÉ PAULISTA.' 01/10/1992 361 f. Mestrado em CIÊNCIA SOCIAL (ANTROPOLOGIA SOCIAL) Instituição de Ensino: UNIVERSIDADE DE SÃO PAULO, São Paulo.

SANTOS, ALESSANDRO DE OLIVEIRA. REPRESENTAÇÕES SOCIAIS DE SAÚDE E DOENÇA NO CANDOMBLÉ JÊJJE-NAGÔ DO BRASIL' 01/11/1999 163 f. Mestrado em PSICOLOGIA ESCOLAR E DO DESENVOLVIMENTO HUMANO Instituição de Ensino: UNIVERSIDADE DE SÃO PAULO, São Paulo.

BERNARDO, ROSA MARIA COSTA. OBJETOS DE AXÉ: UM ESTUDO ANTROPOLOGOGICO QUE VAI DA CONSTRUCAO DO OBJETO A MANUNTENCAO DA VIDA NOS GRUPOS DE CAMDOMBLE DE SAO PAULO.' 01/09/1994 300 f. Mestrado em CIÊNCIA SOCIAL (ANTROPOLOGIA SOCIAL) Instituição de Ensino: UNIVERSIDADE DE SÃO PAULO, São Paulo. 
BARBARA, ROSA MARIA SUSANNA. A DANÇA DO VENTO E DA TEMPESTADE' 01/12/1995 181 f. Mestrado em SOCIOLOGIA Instituição de Ensino: UNIVERSIDADE FEDERAL DA BAHIA, SALVADOR.

ANDREI, ELENA MARIA. COISAS DE SANTO-ICONOGRAFIA DA IMAGINARIA AFROBRASILEIRA' 01/12/1994 293 f. Mestrado em ARTES VISUAIS Instituição de Ensino: UNIVERSIDADE FEDERAL DO RIO DE JANEIRO, RIO DE JANEIRO.

ALFONSO, JOSE LUIS HERNANDEZ. OS DONOS DAS ENCRUZILHADAS' 01/11/1994 114 f. Mestrado em INTEGRAÇÃO DA AMÉRICA LATINA Instituição de Ensino: UNIVERSIDADE DE SÃO PAULO, São Paulo.

SILVA, CARLOS ALBERTO BORGES DA. VALE DOS ORIXAS: ESTUDO SOBRE ACUSAÇÃO DE DEMANDA ENTRE TERREIROS.' 01/03/1994 274 f. Mestrado em ANTROPOLOGIA SOCIAL Instituição de Ensino: UNIVERSIDADE ESTADUAL DE CAMPINAS, CAMPINAS.

FREITAS, RICARDO OLIVEIRA DE. O DIA DO NOME: POR UM CONCEITO DE AFROBRASILIDADE' $\quad 01 / 08 / 1995134$ f. Mestrado em COMUNICAÇÃO Instituição de Ensino: UNIVERSIDADE FEDERAL DO RIO DE JANEIRO, RIO DE JANEIRO.

LUCAS, GLAURA. "OS SONS DO ROSÁRIO - UM ESTUDO ETNOMUSICOLÓGICO DO CONGADO MINEIRO - ARTUROS E JATOBÁ "' 01/04/1999 250 f. Mestrado em ARTES Instituição de Ensino: UNIVERSIDADE DE SÃO PAULO, São Paulo.

MANZOCHI, HELMY MANSUR. "ALGUNS ASPECTOS ICONOGRAFICOS DA PRODUÇÃO PLASTICA RELIGIOSA AFRO-BRASILEIRA".' 01/06/1992 172 f. Mestrado em ARTES (TEATRO CINEMA E ARTES PLASTICAS) Instituição de Ensino: UNIVERSIDADE DE SÃO PAULO, São Paulo.

\section{TESES DO SÉCULO XX}

GIROTO, ISMAEL. O UNIVERSO MÁGICO-RELIGIOSO NEGRO-AFRICANO E AFROBRASILEIRO: BANTU E NAGÓ.' 01/10/1999 412 f. Doutorado em CIÊNCIA SOCIAL (ANTROPOLOGIA SOCIAL) Instituição de Ensino: UNIVERSIDADE DE SÃO PAULO, São Paulo.

HOFBAUER, ANDREAS. UMA HISTÓRIA DE BRANQUEAMENTO OU O NEGRO EM QUESTÃO.' 01/11/1999 250 f. Doutorado em CIÊNCIA SOCIAL (ANTROPOLOGIA SOCIAL) Instituição de Ensino: UNIVERSIDADE DE SÃO PAULO, São Paulo.

VOGEL, ARNO. MUZENZA A METAMORFOSE INICIATICA NACULTURA AFROBRASILEIRA DOS CANDOMBLES' 01/06/1991 291 f. Doutorado em ANTROPOLOGIA SOCIAL Instituição de Ensino: UNIVERSIDADE FEDERAL DO RIO DE JANEIRO, RIO DE JANEIRO.

VIANNA, HELIO. "SOMOS UMA MONTANHA!": ORALIDADE, SOCIEDADE LETRADA E INVENÇÃ̃ DE TRADIÇÕES NO CANDOMBLÉ CARIOCA DO SÉCULO XX.' 01/08/1999 3396 f. Doutorado em ANTROPOLOGIA SOCIAL Instituição de Ensino: UNIVERSIDADE FEDERAL DO RIO DE JANEIRO, RIO DE JANEIRO.

TEIXEIRA, MARIA LINA LEAO. A ENCRUZILHADA DO SER: REPRESENTACOES DA (LOU)CURA EM TERREIROS DE CANDOMBLE.' 01/09/1994 280 f. Doutorado em CIÊNCIA SOCIAL (ANTROPOLOGIA SOCIAL) Instituição de Ensino: UNIVERSIDADE DE SÃO PAULO, São Paulo.

\section{TESES DO SÉCULO XXI}

TESSEROLLI, MIRIAN APARECIDA. SALUBA NANÃ! A VENERÁVEL MÃE ANCESTRAL NA CONTEMPORANEIDADE BRASILEIRA' 18/03/2013 160 f. Doutorado em SOCIOLOGIA E ANTROPOLOGIA Instituição de Ensino: UNIVERSIDADE FEDERAL DO PARÁ, Belém.

Revista da ABPN • v. 12, n. $34 \cdot$ Set - Nov 2020, p. 731-745 
SANTOS, TADEU DOS. O CORPO COMO UM TEXTO VIVO. A FESTA E A DANÇA NO CANDOMBLÉ' 01/08/2003 159 f. Doutorado em CIÊNCIAS DA RELIGIÃO Instituição de Ensino: UNIVERSIDADE METODISTA DE SÃO PAULO, São Bernardo do Campo.

MESQUITA, RALPH RIBEIRO. VIVER E MORRER EM TEMPOS DE AIDS: VIDA, MORTE E SEXUALIDADE EM CANDOMBLÉS DO RIO DE JANEIRO.' 01/05/2002 321 f. Doutorado em SOCIOLOGIA E ANTROPOLOGIA Instituição de Ensino: UNIVERSIDADE FEDERAL DO RIO DE JANEIRO, RIO DE JANEIRO.

CARDOSO, FERNANDA DE SOUZA. GIRANDO EM UMA ROÇA BANTO: A DANÇA COMO ELEMENTO CONSTITUTIVO DO CANDOMBLÉ ANGOLA EM MONTES CLAROS/MG' 17/06/2016 153 f. Doutorado em CIÊNCIA DA RELIGIÃO Instituição de Ensino: PONTIFÍCIA UNIVERSIDADE CATÓLICA DE SÃO PAULO, São Paulo.

PAIVA, KATE LANE COSTA DE. ODARA: UMA ETNOGRAFIA DO GOSTO E DA BELEZA EM UM TERREIRO DE CANDOMBLÉ KETU' 15/12/2014 $226 \mathrm{f}$. Doutorado em ARTES VISUAIS Instituição de Ensino: UNIVERSIDADE FEDERAL DO RIO DE JANEIRO, Rio de Janeiro.

OLIVEIRA, LUCIA MARIA ALVES DE. A DANÇA DOS ORIXÁS E SUAS REPRESENTAÇÕES SOCIAIS NOS CANDOMBLÉS NAGÔ' 30/11/2014 85 f. Doutorado em PSICOLOGIA SOCIAL Instituição de Ensino: UNIVERSIDADE DO ESTADO DO RIO DE JANEIRO, Rio de Janeiro.

QUERETTE, LETICIA LORETO. A TRADIÇÃO XAMBÁ: UM ESTUDO ANTROPOLÓGICO SOBRE O MUSEU SEVERINA PARAÍSO DA SILVA' 02/12/2014 209 f. Doutorado em CIÊNCIAS SOCIAIS Instituição de Ensino: UNIVERSIDADE DO ESTADO DO RIO DE JANEIRO, Rio de Janeiro.

OLIVEIRA, IRIS VERENA SANTOS DE. O FEITIÇO DA CURA. HISTÓRIAS DO POVO DE SANTO, FEITICEIRAS E CURANDEIROS DA BAHIA (1930-1960)' 31/07/2014 297 f. Doutorado em ESTUDOS ÉTNICOS E AFRICANOS Instituição de Ensino: UNIVERSIDADE FEDERAL DA BAHIA, Salvador.

SILVA, RENATO MENDONCA BARRETO DA. A EXPERIÊNCIA BANTU NO RIO DE JANEIRO: O CANDOMBLÉ COMO QUESTÃO ' 21/12/2017 234 f. Doutorado em ARTES VISUAIS Instituição de Ensino: UNIVERSIDADE FEDERAL DO RIO DE JANEIRO, Rio de Janeiro.

SILVA, MARY ANNE VIEIRA. DINÂMICAS TERRITORIAIS DO SAGRADO DE MATRIZ AFRICANA: O CANDOMBLÉ EM GOIÂNIA E REGIÃO METROPOLITANA' 22/04/2013 $258 \mathrm{f}$. Doutorado em GEOGRAFIA Instituição de Ensino: UNIVERSIDADE FEDERAL DE GOIÁS, Goiânia.

JORGE, ERICA FERREIRA DA CUNHA. A INFÂNCIA AFRODESCENDENTE: INICIAÇÃO E SOCIALIZAÇÃO RELIGIOSAS EM UM TERREIRO DE CANDOMBLÉ JEJE-NAGÔ' 21/02/2018 222 f. Doutorado em Ciências Humanas e Sociais Instituição de Ensino: UNIVERSIDADE FEDERAL DO ABC, Santo André.

GAMA, ELIZABETH CASTELANO. LUGARES DE MEMÓRIAS DO POVO-DE-SANTO: PATRIMÔNIO CULTURAL ENTRE MUSEUS E TERREIROS' 09/03/2018 277 f. Doutorado em HISTÓRIA Instituição de Ensino: UNIVERSIDADE FEDERAL FLUMINENSE, Niterói .

PORTUGAL, CLARICE MOREIRA. ENTRE ENREDOS DE AXÉ E REDES DE SAÚDE MENTAL: UMA CARTOETNOGRAFIA DE ADEPTOS DO CANDOMBLÉ EM PROCESSO DE DESINSTITUCIONALIZAÇÃO.' 23/04/2018 undefined f. Doutorado em SAÚDE COLETIVA Instituição de Ensino: UNIVERSIDADE FEDERAL DA BAHIA, Salvador.

MALPASSO, ALESSANDRO. EL TRANCE EN EL XIRÊ: EXPRESIVIDADES DEL CUERPO MEDIANTE UN PROCESO CREATIVO' 16/11/2017 undefined f. Doutorado em DIFUSÃO DO CONHECIMENTO IFBA - SENAI/CIMATEC - LNCC - UNEB - UEFS Instituição de Ensino: UNIVERSIDADE FEDERAL DA BAHIA, Salvador.

Revista da ABPN • v. 12, n. $34 \cdot$ Set - Nov 2020, p. 731-745 
FIGUEIREDO, JANAINA DE. ENTRE PORTOS E RITOS: A MEMÓRIA DO CANDOMBLÉ ANGOLA EM SANTOS' 04/03/2016 215 f. Doutorado em CIÊNCIAS SOCIAIS Instituição de Ensino: PONTIFÍCIA UNIVERSIDADE CATÓLICA DE SÃO PAULO, São Paulo.

PINHO, OSMUNDO ARAÚJO. "O MUNDO NEGRO": SÓCIO-ANTROPOLOGIA DA REAFRICANIZAÇÃO EM SALVADOR' 01/02/2003 412 f. Doutorado em CIÊNCIAS SOCIAIS Instituição de Ensino: UNIVERSIDADE ESTADUAL DE CAMPINAS, CAMPINAS.

LEITE, GILDECI DE OLIVEIRA. EDISON CARNEIRO, BIOGRAFEMAS: POESIA, SAMBA E CANDOMBLÉ' 03/05/2017 440 f. Doutorado em DIFUSÃO DO CONHECIMENTO IFBA SENAI/CIMATEC - LNCC - UNEB - UEFS Instituição de Ensino: UNIVERSIDADE FEDERAL DA BAHIA, Salvador.

TORRES, MARCOS ANTONIO CUNHA. OLHOS BRANCOS SOBRE O SAGRADO NEGRO: A CONSTRUÇÃO DA AFRICANIDADE NAS IMAGENS DE PIERRE VERGER (1902-1996)' 15/05/2015 204 f. Doutorado em HISTÓRIA Instituição de Ensino: UNIVERSIDADE FEDERAL DE GOIÁS, Goiânia.

SANTOS, SILVIA MARIA VIEIRA DOS. JOVEM QUE VELHO RESPEITA - AS EXPERIÊNCIAS E SABERES DA JUVENTUDE CANDOMBLECISTA. ' 02/07/2015 245 f. Doutorado em EDUCAÇÃO Instituição de Ensino: UNIVERSIDADE FEDERAL DO CEARÁ, Fortaleza.

LERRO, LUIZ DANIEL. SCHEMATA CORPORAIS DOS ORIXÁS: AÇÕES, GESTOS E ATITUDES DA CULTURA AFRO-BRASILEIRA NAS METODOLOGIAS DO ENSINO DE TEATRO"' 12/06/2013 undefined f. Doutorado em ARTES CÊNICAS Instituição de Ensino: UNIVERSIDADE FEDERAL DA BAHIA, Salvador.

CORTES, NESTOR GOMES MORA. DO CANDOMBE URUGUAIO: UMA TRAVESSIA PARA ACEITAÇÃO - ETNOPOLÍTICA, CORPOREIDADES E EMANCIPAÇÃO ' 03/02/2016 194 f. Doutorado em ANTROPOLOGIA Instituição de Ensino: UNIVERSIDADE FEDERAL FLUMINENSE, Niterói.

OLIVEIRA, ROBERTO FRANCISCO DE. HIBRIDAÇÃO BANTU: O PERCURSO CULTURAL ADOTADO POR UM POVO.' 29/04/2015 174 f. Doutorado em CIÊNCIAS DA RELIGIÃO Instituição de Ensino: PONTIFÍCIA UNIVERSIDADE CATÓLICA DE GOIÁS, Goiânia.

PEREIRA, MAIRA CONCEICAO ALVES. REDES EDUCATIVAS NO TERREIRO ILÈ OMIDAYÈ: UMA PESQUISA COM OS COTIDIANOS NA CIBERCULTURA' 22/02/2018 $291 \mathrm{f}$. Doutorado em EDUCAÇÃO Instituição de Ensino: UNIVERSIDADE DO ESTADO DO RIO DE JANEIRO, Rio de Janeiro.

CARVALHO, RENATA SIMONI HOMEM DE. BAOBÁ: AYRSON HERÁCLITO E A ÁRVORE DA VIDA' 18/06/2018 317 f. Doutorado em ARTES VISUAIS Instituição de Ensino: UNIVERSIDADE DE BRASÍLIA, Brasília.

BARBARA, ROSAMARIA. A DANÇA DAS AIABÁS: DANÇA, CORPO E COTIDIANO DAS MULHERES DE CANDOMBLÉ' 01/05/2002 211 f. Doutorado em SOCIOLOGIA Instituição de Ensino: UNIVERSIDADE DE SÃO PAULO, São Paulo.

PASSOS, MARLON MARCOS VIEIRA. IYÁ ZULMIRA DE ZUMBÁ: UMA TRAJETÓRIA ENTRE NAÇÕES DE CANDOMBLÉ' 21/10/2016 188 f. Doutorado em ANTROPOLOGIA Instituição de Ensino: UNIVERSIDADE FEDERAL DA BAHIA, Salvador.

SILVA, JOAO PAULO DA. TRÂNSITOS, SABERES E TRADIÇÕES IDENTIDADE, MERCADO RELIGIOSO E TRANSAÇÕES DE (RE)CONHECIMENTO EM UM TERREIRO AFRORELIGIOSO NA CIDADE DE JOÃO PESSOA' 21/06/2017 206 f. Doutorado em CIÊNCIAS SOCIAIS Instituição de Ensino: UNIVERSIDADE FEDERAL DO RIO GRANDE DO NORTE, Natal. 
RIBEIRO, DENIZE DE ALMEIDA. CONCEPÇÕES E ESTRATÉGIAS DE SEGURANÇA ALIMENTAR E NUTRICIONAL ENTRE OS TERREIROS DE CANDOMBLÉ DE NOVOS ALAGADOS/BA.' 30/07/2013 173 f. Doutorado em SAÚDE COLETIVA Instituição de Ensino: UNIVERSIDADE FEDERAL DA BAHIA, Salvador .

DAMASCENO, TATIANA MARIA. NAS ÁGUAS DE IEMANJÁ: UM ESTUDO DAS PRÁTICAS PERFORMATIVAS NO CANDOMBLÉ E NA FESTA À BEIRA-MAR' 26/03/2015 235 f. Doutorado em ARTES CÊNICAS Instituição de Ensino: UNIVERSIDADE FEDERAL DO ESTADO DO RIO DE JANEIRO, Rio de Janeiro.

MARQUES, CARLOS EDUARDO. BANDEIRA BRANCA EM PAU FORTE: A SENZALA DE PAI BENEDITO E O QUILOMBLÉ URBANO DE MANZO NGUNZO KAIANGO' 25/03/2015 $371 \mathrm{f}$. Doutorado em ANTROPOLOGIA SOCIAL Instituição de Ensino: UNIVERSIDADE ESTADUAL DE CAMPINAS, Campinas.

GUIMARAES, LUIZ ERNESTO. TEOLOGIA DA LIBERTAÇÃO E RENOVAÇÃO CARISMÁTICA CATÓLICA: RELIGIÃO E POLÍTICA NA ARQUIDIOCESE DE LONDRINA PR' 07/03/2017 218 f. Doutorado em CIÊNCIAS SOCIAIS Instituição de Ensino: UNIVERSIDADE ESTADUAL PAULISTA JÚLIO DE MESQUITA FILHO ( MARÍLIA ), Marília.

BARBOSA, SILVIA MARIA SILVA. QUE PODER É ESSE? UM ESTUDO DA CONSTITUIÇÃO E DAS RELAÇÕES DE PODER NO ILÊ ASÉ OGUM OMIMKAY Ê ' 03/12/2015 249 f. Doutorado em ESTUDOS INTERDISCIPLINARES SOBRE MULHERES, GÊNERO E FEMINISM Instituição de Ensino: UNIVERSIDADE FEDERAL DA BAHIA, Salvador.

SOARES, DALVA MARIA. "MUITA RELIGIÃO SEU MOÇO!": OS CAMINHOS DE UMA CONGADEIRA' 22/02/2016 171 f. Doutorado em ANTROPOLOGIA SOCIAL Instituição de Ensino: UNIVERSIDADE FEDERAL DE SANTA CATARINA, Florianópolis.

GAMA, LUCIANA BARROS. LAROYÊ! EXU: OS CAMINHOS QUE LEVAM AO MERCADO CONSUMO RELIGIOSO AFRO-BRASILEIRO NO RECIFE - PE' 01/03/2016 159 f. Doutorado em ANTROPOLOGIA Instituição de Ensino: UNIVERSIDADE FEDERAL DE PERNAMBUCO, Recife.

PEREIRA, JAIME SANTANA SODRE. NOTÍCIAS PRODUZIDAS PELA IMPRENSA DA BAHIA SOBRE O CANDOMBLÉ: REFLEXÕES CRÍTICAS (1950 A 1990)' 24/10/2018 231 f. Doutorado em EDUCAÇÃO E CONTEMPORANEIDADE Instituição de Ensino: UNIVERSIDADE DO ESTADO DA BAHIA, Salvador.

DIONISIO, DEJAIR. ENTRE FALOS E FALÁCIAS: PERTENCIMENTOS E DISCURSOS AFROBRASILEIROS NAS NARRATIVAS DE HOMENS EM TOCAIA GRANDE - A FACE OBSCURA, DE JORGE AMADO' 23/05/2014 143 f. Doutorado em LETRAS Instituição de Ensino: UNIVERSIDADE ESTADUAL DE LONDRINA, Londrina.

GORDENSTEIN, SAMUEL LIRA. DE SOBRADO A TERREIRO: A CONSTRUÇÃO DE UM CANDOMBLÉ NA SALVADOR OITOCENTISTA' $12 / 12 / 2014 \quad 361$ f. Doutorado em ANTROPOLOGIA Instituição de Ensino: UNIVERSIDADE FEDERAL DA BAHIA, Salvador.

OLIVEIRA, BRUNO ARAUJO. BARABADA UMA ARQUEOLOGIA DA VIDA E DO FAZER CANDOMBLÉ NA PRISÃO' 29/06/2018 489 f. Doutorado em CIÊNCIAS SOCIAIS Instituição de Ensino: UNIVERSIDADE ESTADUAL DE CAMPINAS, Campinas.

PECHINE, SERGE. INTOLÉRANCE ET RECONNAISSANCE RELIGIEUSE À SALVADOR DE BAHIA. LE FACE-À-FACE DES ÉGLISES NÉOPENTECÔTISTES ET DES RELIGIONS DE MATRICE AFRICAINE EN CE DÉBUT DE XXIE SIÈCLE.' 16/12/2013 $468 \mathrm{f}$. Doutorado em CIÊNCIAS SOCIAIS Instituição de Ensino: UNIVERSIDADE FEDERAL DA BAHIA, Salvador.

SOBRINHO, ANTONIO CARLOS MONTEIRO TEIXEIRA. DAS POSSIBILIDADES HETEROTÓPICAS PARA UMA EXPERIÊNCIA DE LIBERDADE - UM ESTUDO DO 
UNIVERSO FICCIONAL AMADIANO' 08/05/2017 281 f. Doutorado em LITERATURA E CULTURA Instituição de Ensino: UNIVERSIDADE FEDERAL DA BAHIA, .

SOUZA, ELLEN GONZAGA LIMA. EXPERIÊNCIAS DE INFÂNCIAS COM PRODUÇÕES DE CULTURAS NO ILÊ AXÉ OMO OXÉ IBÁ LATAM' 15/02/2016 179 f. Doutorado em EDUCAÇÃO Instituição de Ensino: UNIVERSIDADE FEDERAL DE SÃO CARLOS, São Carlos.

CASALI, RODRIGO. GUIAS E ORIXÁS: NARRATIVAS DE EXPRESSÕES ORAIS SOBRE OS CANDOMBLÉS DO MS' 05/12/2016 405 f. Doutorado em Humanidades, Direitos e outras Legitimidades Instituição de Ensino: UNIVERSIDADE DE SÃO PAULO, São Paulo.

VELAME, FABIO MACEDO. ARQUITETURAS DA VENTURA: OS TERREIROS DE CANDOMBLÉ DE CACHOEIRA E SÃO FÉLIX.' 02/04/2013 250 f. Doutorado em ARQUITETURA E URBANISMO Instituição de Ensino: UNIVERSIDADE FEDERAL DA BAHIA, Salvador.

FLAKSMAN, CLARA MARIANI. NARRATIVAS, RELAÇÕES E EMARANHADOS: OS ENREDOS DO CANDOMBLÉ NO TERREIRO DO GANTOIS, SALVADOR, BAHIA.' 06/06/2014 292 f. Doutorado em ANTROPOLOGIA SOCIAL Instituição de Ensino: UNIVERSIDADE FEDERAL DO RIO DE JANEIRO, Rio de Janeiro.

RAMOS, WELLINGTON SANTOS. "CANDOMBLÉ: RELIGIÃO OU SISTEMA DE SENTINDO TOTALIZANTE DE VIDA? ANÁLISE A PARTIR DOS PENSAMENTOS: DESCOLONIAL E TRADICIONAL AFRICANO"' 03/04/2017 232 f. Doutorado em CIÊNCIAS DA RELIGIÃO Instituição de Ensino: UNIVERSIDADE METODISTA DE SÃO PAULO, São Bernardo do Campo.

TEIXEIRA, VITOR AQUINO DE QUEIROZ DAVILA. A PEDRA QUE RONCA NO MEIO DO MAR: BAIANIDADE, SILÊNCIO E EXPERIÊNCIA RACIAL NA OBRA DE DORIVAL CAYMMI 15/03/2017 234 f. Doutorado em ANTROPOLOGIA SOCIAL Instituição de Ensino: UNIVERSIDADE ESTADUAL DE CAMPINAS, Campinas.

SILVA, GISELIA CASTRO. CULTURA POPULAR AFRO-BRASILEIRA EM EMISSORAS AFILIADAS DA REDE GLOBO : NARRATIVAS AUDIOVISUAIS SOBRE CANDOMBLÉ E TAMBOR DE MINA NA BAHIA E NO MARANHÃO - ANOS 2002/2013' 29/09/2015 undefined f. Doutorado em CULTURA E SOCIEDADE Instituição de Ensino: UNIVERSIDADE FEDERAL DA BAHIA, Salvador.

REIS, LUIZA NASCIMENTO DOS. DE IMPROVISADOS À EMÉRITOS: TRAJETÓRIAS INTELECTUAIS NO CENTRO DE ESTUDOS AFRO-ORIENTAIS (1959-1994)' 25/09/2015 undefined f. Doutorado em ESTUDOS ÉTNICOS E AFRICANOS Instituição de Ensino: UNIVERSIDADE FEDERAL DA BAHIA, Salvador.

MELLO, MARCIO LUIZ BRAGA CORREA DE. PRÁTICAS TERAPÊUTICAS POPULARES E RELIGIOSIDADE AFRO-BRASILEIRA NO RIO DE JANEIRO: UM DIÁLOGO POSSÍVEL ENTRE SAÚDE E ANTROPOLOGIA.' 18/07/2013 136 f. Doutorado em SAÚDE PÚBLICA Instituição de Ensino: FUNDACAO OSWALDO CRUZ, Rio de Janeiro.

MARQUES, ANGELA CRISTINA BORGES. TAMBORES DO SERTÃO: DIFERENÇA COLONIAL E INTERCULTURALIDADE - ENTRELIÇAMENTO ENTRE UMBANDA/QUIMBANDA E CANDOMBLÉ ANGOLA NO NORTE DE MINAS GERAIS' 11/03/2016 304 f. Doutorado em CIÊNCIA DA RELIGIÃO Instituição de Ensino: PONTIFÍCIA UNIVERSIDADE CATÓLICA DE SÃO PAULO, São Paulo.

RIVAS, MARIA ELISE GABRIELE BAGGIO MACHADO. TEM MULHER NA MACUMBA "SIM SINHÔ": AS MULHERES NEGRAS NA MACUMBA RELIGIOSA E MUSICAL CARIOCA ENTRE 1870 E 1930' 17/11/2017 265 f. Doutorado em CIÊNCIA DA RELIGIÃO Instituição de Ensino: PONTIFÍCIA UNIVERSIDADE CATÓLICA DE SÃO PAULO, São Paulo.

LIRA, LILIAN CONCEICAO DA SILVA PESSOA DE. ELEMENTOS TEOPEDAGÓGICOS AFROCENTRADOS PARA SUPERAÇÃO DA VIOLÊNCIA DE GÊNERO CONTRA AS

Revista da ABPN • v. 12, n. $34 \cdot$ Set - Nov 2020, p. 731-745 
MULHERES NEGRAS: DIÁlOGO COM A COMUNIDADE-TERREIRO ILÉ ÀṢE YEMỌJÁ OMI OLODÒ E 'O ACOLHIMENTO QUE ALIMENTA A ANCESTRALIDADE.' 21/03/2014 244 f. Doutorado em TEOLOGIA Instituição de Ensino: ESCOLA SUPERIOR DE TEOLOGIA, São Leopoldo.

NUNES, ERIVALDO SALES. CONTRIBUIÇÃO PARA A HISTÓRIA DO CANDOMBLÉ CONGO-ANGOLA NA BAHIA O TERREIRO DE BERNARDINO DO BATE FOLHA (19161946) ' 08/03/2017 384 f. Doutorado em HISTÓRIA Instituição de Ensino: UNIVERSIDADE FEDERAL DA BAHIA, Salvador.

NIEL, MARCELO MAXIMO. ÀWỌN ‘ONÀ MÍMọ́: OS CAMINHOS SAGRADOS DAS MULHERES DO AXÉ EM NOVA IORQUE(EUA)' 01/12/2017 109 f. Doutorado em SAÚDE COLETIVA Instituição de Ensino: UNIVERSIDADE FEDERAL DE SÃO PAULO, São Paulo Biblioteca.

MENEZES, CLAUDIA CASTELLANO DE. LUGAR DE ORUM E AYÊ; AMBIÊNCIA, CONFLITO E DINÂMICAS DE APROPRIAÇÃO DO CANDOMBLÉ NO ESPAÇO URBANO PÚBLICO.' 29/03/2017 208 f. Doutorado em ARQUITETURA Instituição de Ensino: UNIVERSIDADE FEDERAL DO RIO DE JANEIRO, Rio de Janeiro.

CORDEIRO, MARIA DA CONCEICAO DA SILVA. "DOENÇA DE FEITIÇO", AÇÕES TERAPÊUTICAS E OS PERCURSOS DE CURA NOS TERREIROS DE UMBANDA E CANDOMBLÉ EM MACAPÁ-AP. ' 20/12/2016 undefined f. Doutorado em SOCIOLOGIA Instituição de Ensino: UNIVERSIDADE FEDERAL DO CEARÁ, Fortaleza.

SOUZA, WALDELICE MARIA SILVA DE. A ROTAÇÃO DAS IDENTIDADES: O TRANSE COMO DÍNAMO DA ARQUITETURA DAS PERSONALIDADES NOS ORÍKİS E DOS PERSONAGENS NA OBRA DE PEPETELA' 13/02/2014 459 f. Doutorado em LETRAS (CIÊNCIA DA LITERATURA) Instituição de Ensino: UNIVERSIDADE FEDERAL DO RIO DE JANEIRO, Rio de Janeiro.

SANTOS, FRANCISCO WELLINGTON PARA DOS. BRINCANDO NO (CANTO-QUE-DANÇA) DO IJEXÁ COM O BUMBA-MEU-BOI: TEATRO DO ENCANTAMENTO DA ANCESTRALIDADE AFRICANA EM FORTALEZA A CIDADE TAN-TAN.' 10/07/2015 $270 \mathrm{f}$. Doutorado em EDUCAÇÃO Instituição de Ensino: UNIVERSIDADE FEDERAL DO CEARÁ, Fortaleza.

CONCEICAO, SUELI SANTOS. CARTOGRAFIA SOCIAL DOS TERREIROS DE CANDOMBLÉ DA REGIÃO METROPOLITANA DE SALVADOR, BA: RISCOS, LIMITES, DESAFIOS À LONGEVIDADE INSTITUCIONAL DOS RITUAIS SAGRADOS' 08/03/2018 $160 \mathrm{f}$. Doutorado em DESENVOLVIMENTO E MEIO AMBIENTE UFPI-UFRN-FUFSE-UESC-UFPB/JP Instituição de Ensino: UNIVERSIDADE ESTADUAL DE SANTA CRUZ, Recife.

VALE, MAIRA CAVALCANTI. CACHOEIRA \& A INVERSÃO DO MUNDO' 04/10/2018 269 f. Doutorado em ANTROPOLOGIA SOCIAL Instituição de Ensino: UNIVERSIDADE ESTADUAL DE CAMPINAS, Campinas.

JESUS, ERICIVALDO VEIGA DE. A COZINHA BAIANA DO RESTAURANTE ESCOLA DO SENAC DO PELOURINHO, BAHIA: MUDANÇAS DE CONTEXTO E ATORES' 01/11/2002 252 f. Doutorado em CIÊNCIAS SOCIAIS Instituição de Ensino: PONTIFÍCIA UNIVERSIDADE CATÓLICA DE SÃO PAULO, SÃO PAULO.

COSTA, MONICA SILVA DA. DANÇAS E HISTÓRIAS DE OSUN, YEMOJÁ E AZILI ENTRE O BENIN E O BRASIL: TRADIÇÕES, TRANSMISSÕES E REGISTROS CORPORAIS.' 15/05/2015 300 f. Doutorado em ARTES CÊNICAS Instituição de Ensino: UNIVERSIDADE FEDERAL DO ESTADO DO RIO DE JANEIRO, Rio de Janeiro.

SILVA, GERANILDE COSTA E. PRETAGOgIA: CONSTRUINDO UM REFERENCIAL TEÓRICO-METODOLÓGICO, DE BASE AFRICANA, PARA A FORMAÇÃO DE PROFESSORES/AS' 12/07/2013 243 f. Doutorado em EDUCAÇÃO Instituição de Ensino: UNIVERSIDADE FEDERAL DO CEARÁ, Fortaleza. 
RIBEIRO, OBERTAL XAVIER. - AXIOLOGIA - VALORES ÉTNICORRACIAIS, CULTURAIS E RELIGIOSOS UMA ABORDAGEM EM PRÁTICAS EDUCATIVAS' 19/07/2018 299 f. Doutorado em TEOLOGIA Instituição de Ensino: ESCOLA SUPERIOR DE TEOLOGIA, São Leopoldo.

BANDEIRA, LUIS CLAUDIO CARDOSO. "ROTAS E RAIZES" DE ANCESTRAIS ITINERANTES' 03/06/2013 291 f. Doutorado em HISTÓRIA Instituição de Ensino: PONTIFÍCIA UNIVERSIDADE CATÓLICA DE SÃO PAULO, São Paulo.

RESTREPO, DARIO HENAO. O Código XANGô. A COSMOVISÃo MITO-POÉTICA DE MATRIZ AFRICANA EM CHANGÓ, EL GRAN PUTAS DE MANUEL ZAPATA OLIVELLA' 03/06/2016 216 f. Doutorado em LETRAS NEOLATINAS Instituição de Ensino: UNIVERSIDADE FEDERAL DO RIO DE JANEIRO, Rio de Janeiro.

RAMOS, MARIA ESTELA ROCHA. BAIRROS NEGROS: UMA LACUNA NOS ESTUDOS URBANÍSTICOS UM ESTUDO EMPÍRICO-CONCEITUAL NO BAIRRO DO ENGENHO VELHO DA FEDERAÇÃO, SALVADOR (BAHIA).' 05/07/2013 283 f. Doutorado em ARQUITETURA E URBANISMO Instituição de Ensino: UNIVERSIDADE FEDERAL DA BAHIA, Salvador.

CRUZ, NORVAL BATISTA. CORPO, ANCESTRALIDADE, ORALIDADE E EDUCAÇÃO NO ELÊ AXÉ OMO TIFÉ - O CORPO DE XANGÔ' 27/09/2013 151 f. Doutorado em EDUCAÇÃO Instituição de Ensino: UNIVERSIDADE FEDERAL DO CEARÁ, Fortaleza.

AFONSO, CAMILO. A EDUCAÇÃO TRADICIONAL DO NOROESTE DE ANGOLA:FORMAS DE TRANSMISSÃO DE SABERES E SUA PRESENÇA NA BAHIA.' 22/04/2016 303 f. Doutorado em EDUCAÇÃO E CONTEMPORANEIDADE Instituição de Ensino: UNIVERSIDADE DO ESTADO DA BAHIA, Salvador.

MELO, RAFAEL JOSE DE. GRAMÁTICA DO BANQUETE DOS ORIXÁS: MEMÓRIA DAS VOZES' 15/12/2014 594 f. Doutorado em LINGÜÍSTICA Instituição de Ensino: UNIVERSIDADE FEDERAL DA PARAÍBA/JOÃO PESSOA, João Pessoa.

PIMENTEL, CLAUDIO SANTANA. ALMAS LADINAS: AS MUITAS ÁFRICAS DE ANTONIO OLINTO E SUA CONTRIBUIÇÃO AO ESTUDO DAS RELIGIÕES' 12/02/2014 157 f. Doutorado em CIÊNCIA DA RELIGIÃO Instituição de Ensino: PONTIFÍCIA UNIVERSIDADE CATÓLICA DE SÃO PAULO, São Paulo.

CUNHA, WELTHON RODRIGUES. TRANSE MEDIÚNICO: ENTRE A CIÊNCIA E A RELIGIÃO.' 18/02/2013 201 f. Doutorado em CIÊNCIAS DA RELIGIÃO Instituição de Ensino: PONTIFÍCIA UNIVERSIDADE CATÓLICA DE GOIÁS, Goiânia.

SILVA, MARIANA DE LIMA E. OMÓ MIMÓ, O FILHO DO AMOR: UM ESTUDO SOBRE OS FILHOS DE LOGUN EDÉ' 08/03/2013 276 f. Doutorado em ANTROPOLOGIA Instituição de Ensino: UNIVERSIDADE DE BRASÍLIA, Brasília.

PEDREIRA, CAROLINA SOUZA. TECIDOS DO MUNDO: ALMAS, ESPÍRITOS E CABOCLOS EM ANDARAÍ, BAHIA.' 26/02/2015 2016 f. Doutorado em ANTROPOLOGIA Instituição de Ensino: UNIVERSIDADE DE BRASÍLIA, Brasília.

BASSO, JORGE GARCIA. AGENOR MIRANDA ROCHA: UM PROFESSOR ENTRE DOIS MUNDOS' 26/02/2016 211 f. Doutorado em EDUCAÇÃO: HISTÓRIA, POLÍTICA, SOCIEDADE Instituição de Ensino: PONTIFÍCIA UNIVERSIDADE CATTÓLICA DE SÃO PAULO, São Paulo.

SILVA, FLORIANA ROSA DA. A CACHAÇA NOS RITUAIS AFRO-BRASILEIROS: CANTOS, CRENÇAS E CURAS EM TERREIROS DE UMBANDA UBERLÂNDIA - MG (2012 - 2017) .' 02/07/2018ndefined f. Doutorado em HISTÓRIA Instituição de Ensino: UNIVERSIDADE FEDERAL DE UBERLÂNDIA, Uberlândia. 
RODRIGUES, MICHELLE GONCALVES. DA INVISIBILIDADE À VISIBILIDADE DA JUREMA: A RELIGIÃO COMO POTENCIALIDADE POLÍ́TICA' 23/05/2014 201 f. Doutorado em ANTROPOLOGIA Instituição de Ensino: UNIVERSIDADE FEDERAL DE PERNAMBUCO, Recife.

MARCELO, JOAO RANGEL. LOUVADO SEJA! MARCAS DE UMA RELIGIOSIDADE LATINOAMERICANA' 31/10/2016 166 f. Doutorado em INTEGRAÇÃO DA AMÉRICA LATINA Instituição de Ensino: UNIVERSIDADE DE SÃO PAULO, São Paulo.

BARRETO, HELEN SABRINA GLEDHILL. TRAVESSIAS RACIALISTAS NO ATLÂNTICO NEGRO: REFLEXÕES SOBRE BOOKER T. WASHINGTON E MANUEL R. QUERINO' 19/03/2014 302 f. Doutorado em ESTUDOS ÉTNICOS E AFRICANOS Instituição de Ensino: UNIVERSIDADE FEDERAL DA BAHIA, Salvador.

SANTOS, MILTON SILVA DOS. RELIGIÃO E DEMANDA: O FENÔMENO RELIGIOSO EM ESCOLAS PÚBLICAS CAMPINAS-SP 2016' 29/09/2016 237 f. Doutorado em ANTROPOLOGIA SOCIAL Instituição de Ensino: UNIVERSIDADE ESTADUAL DE CAMPINAS, Campinas.

OLIVEIRA, ROSENILTON SILVA DE. A COR DA FÉ: “IDENTIDADE NEGRA” E RELIGIÃO' 02/03/2017 262 f. Doutorado em CIÊNCIA SOCIAL (ANTROPOLOGIA SOCIAL) Instituição de Ensino: UNIVERSIDADE DE SÃO PAULO, São Paulo.

MOURA, ADRIANO CARLOS DE. O ESPETÁCULO SEMIÓTICO DO MARACATU RURAL DA ZONA DA MATA NORTE PERNAMBUCANA' 21/03/2016 227 f. Doutorado em LETRAS Instituição de Ensino: UNIVERSIDADE FEDERAL DA PARAÍBA ( JOÃO PESSOA ), João Pessoa.

GARCIA, CELIO DE PADUA. EM TERRAS DE SINCRETISMOS: APROPRIAÇÕES E RESSIGNIFICAÇÕES AFRO-BRASILEIRAS NA IGREJA UNIVERSAL DO REINO DE DEUS.' 29/06/2015 191 f. Doutorado em CIÊNCIAS DA RELIGIÃO Instituição de Ensino: PONTIFÍCIA UNIVERSIDADE CATÓLICA DE GOIÁS, Goiânia.

FILHO, JOAO SIMOES CARDOSO. "DANÇANDO PRA IEMANJÁ" - RETORNANDO AO FESTIVAL DE IEMANJÁ: 1995 a 2013. DINÂMICAS SOCIAIS, POLÍTICAS E RELIGIOSAS, NUMA PERSPECTIVA ANTROPOLÓGICA E UM OLHAR HERMENÊUTICO' 05/10/2015 $242 \mathrm{f}$. Doutorado em SOCIOLOGIA E ANTROPOLOGIA Instituição de Ensino: UNIVERSIDADE FEDERAL DO PARÁ, Belém.

ALVES, TANIZE TOMASI. O COMPORTAMENTO RELACIONAL NOS PROCESSOS REGIONALIZADORES COTIDIANOS: UM ESTUDO COMPARATIVO ENTRE OS QUILOMBOLAS DE SÃO JOÃO E SANTA CRUZ - PR.' 03/04/2017 190 f. Doutorado em GEOGRAFIA Instituição de Ensino: UNIVERSIDADE FEDERAL DO PARANÁ, Curitiba.

NASCENTES, ZAMA CAIXETA. MAGIA RELIGIÃO E CIÊNCIA EM CORPO DE BAILE' 17/12/2013 592 f. Doutorado em LETRAS Instituição de Ensino: UNIVERSIDADE FEDERAL DO PARANÁ, Curitiba.

MOREIRA, TIAGO DE ALMEIDA. O ESPAÇO PÚBLICO DA CIDADE DE SALVADOR/BA NO CINEMA DO INÍCIO DO SÉCULO XXI' 06/11/2017 226 f. Doutorado em GEOGRAFIA Instituição de Ensino: UNIVERSIDADE DE BRASÍLIA, Brasília.

MENDES, DULCE SANTORO. COM UM TE BOTARAM COM DOIS EU TE TIRO! UM ESTUDO SOBRE AS BENZEDEIRAS E DOS BENZEDEIROS MORADORES DAS COMUNIDADES QUILOMBOLAS DE IGREJA NOVA - ALAGOAS' 29/11/2017 313 f. Doutorado em PSICOSSOCIOLOGIA DE COMUNID.E ECOLOGIA SOCIAL Instituição de Ensino: UNIVERSIDADE FEDERAL DO RIO DE JANEIRO, Rio de Janeiro.

OLIVEIRA, ORLANDO JOSE RIBEIRO DE. O MERCADO DAS FOLHAS NA PEDRA: PRODUÇÃO E CIRCULAÇÃO DE PLANTAS RITUAIS/MEDICINAS NA FEIRA DE SÃO JOAQUIM, SALVADOR(BA)' 29/05/2017 225 f. Doutorado em CIÊNCIAS SOCIAIS Instituição de Ensino: UNIVERSIDADE FEDERAL DA BAHIA, Salvador. 
NASCIMENTO, ALINE SANTOS DE BRITO. TRADIÇÃO, TRADUÇÃO, HIBRIDISMO E RESISTÊNCIA DA IDENTIDADE AFROBRASILEIRA NA LITERATURA AMADIANA' 25/09/2017 197 f. Doutorado em LETRAS Instituição de Ensino: UNIVERSIDADE FEDERAL DO ESPÍRITO SANTO, Vitória.

FREITAS, CILMA LAURINDA. MITOLOGIA DOS ORIXÁS E UMBANDA: DUAS BACIAS SEMÂNTICAS NA PERSPECTIVA DE DURAND.' 20/12/2016 307 f. Doutorado em CIÊNCIAS DA RELIGIÃO Instituição de Ensino: PONTIFÍCIA UNIVERSIDADE CATÓLICA DE GOIÁS, Goiânia.

MEIRA, CELIO SILVA. A TERRITORIALIZAÇÃO DA UMBANDA NA CIDADE DE POÇÕESBA ' 21/09/2018 $181 \mathrm{f}$. Doutorado em PLANEJAMENTO TERRITORIAL E DESENVOLVIMENTO SOCIAL Instituição de Ensino: UNIVERSIDADE CATÓLICA DO SALVADOR, Salvador.

MARTINS, ALESSANDRA RIBEIRO. MATRIZ AFRICANA EM CAMPINAS: TERRITÓRIOS, MEMÓRIA E REPRESENTAÇ̃̃O' 06/02/2017 295 f. Doutorado em Arquitetura e Urbanismo Instituição de Ensino: PONTIFÍCIA UNIVERSIDADE CATÓLICA DE CAMPINAS, Campinas.

FILHO, JOSE HONORIO DAS FLORES. BROTOPENTACOSTALISMO: A RELIGIOSIDADE NEGRA DA PERIFERIA BRASILEIRA - UM ESTUDO DA PERIFERIA URBANA DA REGIÃO METROPOLITANA DE JOÃO PESSOA ' 13/04/2017 245 f. Doutorado em CIÊNCIAS DA RELIGIÃO Instituição de Ensino: UNIVERSIDADE METODISTA DE SÃO PAULO, São Bernardo do Campo.

MACHADO, FELIPE WIRCKER. DANÇA E RITUAL: DO SAGRADO AO CÊNICO' 27/04/2018 230 f. Doutorado em LITERATURA, CULTURA E CONTEMPORANEIDADE Instituição de Ensino: PONTIFÍCIA UNIVERSIDADE CATÓLICA DO RIO DE JANEIRO, Rio de Janeiro.

\section{DÁRÓ 5}

Conclui-se que, das 30 dissertações e 100 teses compiladas do século XX e XXI, no século XX houve uma produção e ascensão de 30 dissertações e 5 teses que comtemplam a temática explicitada nesse texto, e outro ponto a ser considerado, é o crescimento expressivo de escritos no século XXI que totalizaram 95 teses.

É importante reafirmar que $80,77 \%$ dessas pesquisas foram desenvolvidas em Instituições de Ensino Superior (IES) Públicas. Analisando as dissertações do século XX, a região Sudeste do país foi responsável por $90 \%$ das produções, seguida pelo Nordeste com $6,6 \%$ e o Centro-Oeste com $3,4 \%$. Em relação as teses do século XX, todas elas desenvolvidas em IES públicas e na região Sudeste do Brasil.

Sobre as teses do século XXI, as IES públicas foram responsáveis por $81,6 \%$ das pesquisas produzidas, e as regiões geográficas que mais produziram sobre a temática foi a região Sudeste e Nordeste.

Dessa forma, conclui-se que mesmo com o número ainda que simplório, para as pesquisas relacionadas à temática do trabalho, há uma legislação que garante a inserção

\footnotetext{
${ }^{5}$ Palavra em iorubá que significa refletir.
} 
e ensino da cultura africana, afro-brasileira e indígena por meio dos dispositivos legais 10.639/2003 e 11.645/2008. (Brasil, 2008). E acredita-se que foi por meio dessa legislação que houve o aumento de estudos relacionados às religiões afro-brasileiras, tendo em vista que, toda essa conquista foi, e é fruto da luta do Movimento Negro no Brasil.

\section{REFERÊNCIAS BIBLIOGRÁFICAS}

BRASIL. Lei 10.639/2003, de 9 de janeiro de 2003. Altera a Lei n ${ }^{\circ}$ 9. 394, de 20 de dezembro de 1996. Diário Oficial da União, Poder Executivo, Brasília.

BRASIL. Lei 11.645/08 de 10 de março de 2008. Diário Oficial da União, Poder Executivo, Brasília.

MACEDO, Yuri Miguel; OLIVEIRA, Eduardo David; PEÇANHA, Cinézio Feliciano. A CAPOEIRA COMO REFERENCIAL METODOLÓGICO PARA ENFRENTAMENTO DO RACISMO. Revista da Associação Brasileira de Pesquisadores/as Negros/as (f), [S.l.], v. 11, n. Ed. Especi, p. 174-187, out. 2019. ISSN 2177-2770. Disponível em: <http://abpnrevista.org.br/revista/index.php/revistaabpn1/article/view/699>. Acesso em: 05 nov. 2019.

PASSOS, Ana Helena Ithamar. Um estudo sobre branquitude no contexto de reconfiguração das relações raciais no Brasil, 2003-2013. 1.ed. Porto Seguro: Editora Oyá, 2019, 180 p.

SANTOS, Mariana Fernandes dos; VALADARES, Flavio Biasutti; MACEDO, Yuri Miguel. (Des)encontros para um currículo afrocentrado no ensino de língua portuguesa na educação profissional técnica de nível médio integrada ao Ensino Médio. Revista Exitus, v. 9, p. 204-231, 2019. Disponível em: 〈http://dx.doi.org/10.24065/2237-9460.2019v9n4ID1010〉. Acesso em: 05 nov. 2019.

Recebido 14/02/2020

Aprovado em 01/11/2020 